\title{
The Mutual Domestication of Users and Algorithmic Recommendations on Netflix
}

\author{
Ignacio Siles ${ }^{D}$, Johan Espinoza-Rojas, Adrián Naranjo \& \\ María Fernanda Tristán \\ School of Communication, Universidad de Costa Rica AP 2060, San Jose, Costa Rica
}

\begin{abstract}
This article examines the mutual domestication of users and recommendation algorithms on Netflix. Based on 25 interviews with users and an inductive analysis of their practices and profiles on the platform, we discuss five dynamics through which this mutual domestication occurs: personalization, or the ways in which individualized relationships between users and the platform are built; how algorithmic recommendations are integrated into a matrix of cultural codes; the rituals through which they are incorporated into spatial and temporal processes in daily life; the resistance to various aspects of Netflix as a form to enact agency; and the conversion or transformation of the private consumption of the platform into a public issue. The conclusion elaborates on the theoretical and analytical implications of this approach, to rethink the relationship between algorithms and culture.
\end{abstract}

Keywords: Algorithms, Audiences, Culture, Domestication, Latin America, Netflix, Users,

doi:10.1093/ccc/tcz025

Algorithms have reached a privileged status in the contemporary digital ecology. However, various authors have expressed concerns about the rise of algorithmic procedures in the daily lives of their users. Couldry and Mejias (2019, p. 337), for example, argued that algorithms are a key in enacting "data colonialism," a new form of exploitation that "combines the predatory extractive practices of historical colonialism with the abstract quantification methods of computing." At stake in the establishment of algorithmic recommendation systems are the conditions for the redefinition of subjectivity and culture itself.

Despite its many analytical contributions, work on these issues has been limited by its focus on algorithms themselves or their production processes, rather than how users relate to them in everyday life. We know that certain cultural concepts are inscribed in algorithms and that companies use their opacity to establish commercial objectives. However, we understand less well how users incorporate them (or not)

Corresponding author: Ignacio Siles; e-mail: ignacio.siles@ucr.ac.cr 
into their practices as audiences, and what the consequences of these interactions are. This task is challenging, given that algorithms operate invisibly as part of technological assemblages that make their functioning and influences difficult to determine. This study faces this challenge by analyzing how a group of users relate to Netflix's recommendation algorithms.

We argue that the relationship between users and algorithms may be framed in terms of "mutual domestication": users incorporate algorithmic recommendations into everyday life as much as the platform works to colonize users and turn them into ideal consumers through its algorithms. We discuss five specific mechanisms through which this process takes place: personalization, or the ways in which individualized relationships between users and the platform are built; how algorithmic recommendations are integrated into a matrix of cultural codes; the rituals through which they are incorporated into spatial and temporal processes of daily life; the resistance to various aspects of Netflix; and the conversion of these algorithmic recommendations into ways of participating in public life. In this way, we provide empirical specificity to recent work on the role of audiences in processes of datification (Bucher, 2017; Eslami et al., 2015; Livingstone, 2019).

Mutual domestication needs to be understood as cyclical: that is, each one of its dynamics depend on and feed each other (Silverstone, 1994, p. 124). In this way, it becomes possible to further recognize two cyclical, simultaneous processes. On the one hand, algorithms participate in the creation and maintenance of contemporary culture: that is, the "particular way[s] of life, which [express] certain meanings and values not only in art and learning but also in institutions and ordinary behavior" (Williams, 2001, p. 57). On the other hand, algorithms do not work entirely alone: they need to be enacted culturally as they are incorporated into daily lives (Seaver, 2017). Thus, it is necessary to account not only for how algorithms shape culture but also for how algorithms are culture in themselves. The use of algorithms thus becomes a privileged space to examine these processes empirically.

Although our interest is in algorithms, we argue that it is impossible to disassociate how users relate to them from how they work to domesticate Netflix as a platform. This is because they are both part of what Finn (2017, p. 2) calls "culture machines": assemblages of algorithms, platforms, and people. Recommendation algorithms are central in the Netflix culture machine. According to the company's own estimation, "75\% of what people watch is from some sort of recommendation" (Amatriain \& Basilico, 2012a). In this context, platforms are the means through which users attempt to domesticate the opaqueness of algorithms.

We also contribute to research on algorithms and user engagement by conducting empirical work in Costa Rica. The focus on Latin America is relevant, given current discussions of "cultural imperialism" that surround Netflix (Lobato, 2019). Costa Rica illustrates Netflix's specific interest in Latin America: it has a relatively large middle-class, reliable telecommunications infrastructure, and high Internet access rates (Gao, 2015; Red 506, 2018). Finally, we argue that current claims about "data colonialism" warrant a closer look at how exploitation practices associated with 
computing methods are experienced in the places where historical colonialism took place. This could help provide an empirical test for Couldry and Mejias' (2019, p. 337) assertion that "North-South, East-West divisions no longer matter in the same way."

\section{The domestication of media technologies}

The notion of domestication integrates multiple approaches to understand how users incorporate media technologies into their daily lives (Siles \& Boczkowski, 2012). The concept comes mainly from the founding work of Roger Silverstone and his collaborators (Silverstone, 2006). Referring specifically to the case of television, Silverstone (1994, p. 98) defined domestication as "the capacity of a social group ... to appropriate technological artefacts and delivery systems into its own cultureits own spaces and times, its own aesthetic and its own functioning." Silverstone emphasized the "double articulation" that underlies every process of domestication: media technologies are both cultural artifacts and carriers of cultural meaning.

For Silverstone (1994), domestication takes place through several dynamics: (a) appropriation, as the technology is sold and a user becomes its owner; (b) objectification, as the technology acquires physical disposition in the spatial environment of a household; (c) incorporation, as the technology is incorporated into temporal routines in daily life; and (d) conversion, as the technology configures relationships between users and the world outside the household.

More recent work (including Silverstone's own writings) has extended this approach in three significant ways: by taking the domestication process outside the domestic sphere; by endowing it with social, symbolic, and cognitive components; and by defining domestication as a circular process with multiple entry points, enacted by people in various ways. We argue that the rise of streaming platforms offers an opportunity to extend domestication research in productive ways. As Lobato (2019, p. 101; emphasis in original) notes, "Netflix is not a unitary thing but a complex and dynamic metasystem made up of hundreds of different software processes that relies on hard and soft technical infrastructures, open and closed knowledge systems, and public and private investment." Netflix has also pursued differentiated strategies around the globe to become the new standard of transnational television. Taken together, these developments point to significant transformations in the "double articulation" of Internet-distributed television. Algorithms sit at the intersection of these reconfigurations.

\section{Research design}

This study drew on in-depth interviews with 25 Netflix users in Costa Rica to identify the domestication dynamics of the platform. We specifically sought people who defined themselves as heavy Netflix users. This allowed us to focus on individuals who spend significant time using the platform and whose relationship with it is 
highly reflexive: that is, they devote time trying to understand how Netflix operates. We shared a call for participation on social media and selected 20 individuals with different profiles among those who responded. Given our focus on heavy users, most early respondents came from the communications industry. To balance our sample of informants, we asked interviewees for additional suggestions of Netflix users with different backgrounds. The final sample included mostly educated people with a diversity of professional backgrounds who were at different stages in their careers. The age of interviewees ranged between 20 and 53 years old. The final sample also reflected a balance between men (52\%) and women (48\%), to reflect patterns identified in existing studies in the country (Red 506, 2018). All interviews were conducted in person (between January 2017 and February 2018) and lasted an average of 53 minutes. Most conversations took place in the School of Communication at the University of Costa Rica. Interviews were recorded and transcribed in their entirety. We used pseudonyms to protect the identity of our interviewees. (Interviews were conducted in Spanish. All translations are our own.)

To conduct the interviews, we used an adapted version of the "think aloud protocol." In this way, we sought to implement the tactic suggested by Seaver (2017, p. 7), of "treating interviews as fieldwork." We requested data about the practices and trajectories of each person's Netflix use. We asked interviewees to open their account on a computer, which was projected on a screen so that the research team could simultaneously see the content available and the ways in which interviewees interacted with the platform. We asked informants to describe the technological and content particularities of their accounts. We also asked them to reproduce typical appropriation behaviors, discussed specific examples of the content available, and requested explanations of the status of their accounts.

Some research shows that most users are unaware of algorithms and their workings (Eslami et al., 2015). Consistently, few people interviewed for this study used the term "algorithm" or assigned specific activities to its functioning. Yet, forming hypotheses about how recommendations work is a core activity in using digital media. Users believe they understand the logic of the recommendation process and assume that their assumptions are correct. We thus interrogated these hypotheses-or "folk theories"-as a key component of "algorithmic imaginaries": that is, "ways of thinking about what algorithms are, what they should be, how they function and what these imaginations in turn make possible" (Bucher, 2017, pp. 39-40). These imaginaries become productive sites to examine how users relate to opaque entities that work to integrate them into the Netflix system and how they gain specific senses of agency through this interaction with algorithms.

We captured screenshots constantly for the purpose of analyzing them. This made it possible to triangulate data sources, such as verbal descriptions of the interviewees, images, and texts available on the accounts of users. Then, we compared accounts from users with descriptions of how Netflix's algorithmic recommendations work that were provided by the company's representatives in the mainstream media and official outlets. 
Grounded theory allowed us to transform the data into a set of analytical constructs that explain the patterns we identified (Corbin \& Strauss, 2008). We carried out open and axial coding individually to identify data patterns, as well as relationships between the patterns. Five dynamics of mutual domestication were identified in these stages. The research team then conducted selective coding collectively to flesh out these five categories: that is, to develop their main properties into theoretical constructs. The framework of mutual domestication was articulated in the process of selective coding.

\section{The mutual domestication of users and algorithms on Netflix}

Netflix was launched in Costa Rica in September 2011 as part of early attempts to find new users beyond North America. Its use has grown significantly since then. A recent study considered Netflix as among the most-used entertainment apps in the country (Red 506, 2018). According to this study, Netflix ranked high among applications that users in Costa Rica deemed "they could not live without." The platform is used almost equally by people who identify themselves as men (53\%) and women (47\%). Like in the United States, three plans can be acquired in the country: basic ( $\$ 8.99$ per month); standard (\$12.99 per month); and premium (\$15.99 per month), which allows more screens to be simultaneously connected to the service and better image quality. As Lobato (2019) showed, Netflix's relatively uniform global rate doesn't mean that access to the platform is distributed equally across the world. In Costa Rica, the service has been welcomed primarily by the middle and upper classes (Red $506,2018)$.

Netflix is widely integrated into the daily lives of its users. Most interviewees said they dedicate at least 1 hour a day (on weekdays) to watching content and many more hours on weekends. Netflix is a part of a wider entertainment ecology, in which streaming and downloading applications are common. Many interviewees said they have decreased the consumption of traditional television or even abandoned it altogether, but continue to download content through torrent applications. The domestication of Netflix is carried out through a variety of technologies, including television (connected to the Internet, computers, and devices such as Apple TV), cell phones, and tablets.

The mutual domestication of users and recommendations on Netflix can be conceptualized as a cyclical process marked by five dynamics: personalization, integration, ritual, resistance, and conversion. If algorithms are culture, as Seaver (2017) contends-that is, consequences of human practices-we consider these five dynamics as methods that users employ to enact recommendation algorithms in practice.

\section{Personalization}

Personalization refers to the efforts dedicated to building an individualized relationship between users and Netflix. Through personalization, users turn their profile on 


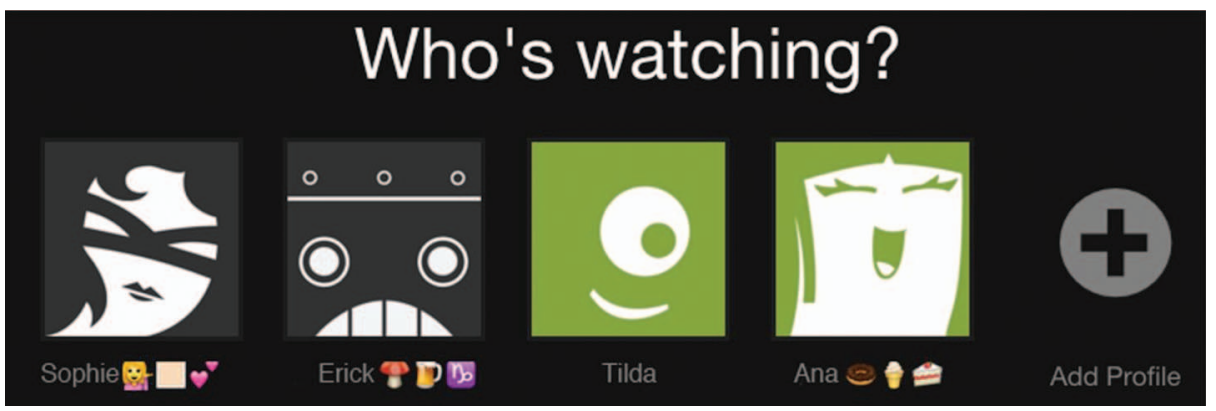

Figure 1 Profiles in Erick's account.

the platform into a technological reflection of their personality, and Netflix uses the inputs provided through these profiles to offer further recommendations (and thus domesticate them).

Netflix has crafted a public identity as a company that specializes in personalization. As Netflix chief content officer, Ted Sarandos, put it, "our brand is really about personalization" (cited in Rohit, 2014). The work of personalization is accomplished in a variety of ways: "at Netflix, we embrace personalization and algorithmically adapt many aspects of our member experience, including the rows we select for the homepage, the titles we select for those rows, the galleries we display, the messages we send, and so forth" (Chandrashekar, Amat, Basilico, \& Jebara, 2017). The company has emphasized the idea that it does not offer a single product for all users, but rather "over a 100 million different products with one for each of our members with personalized recommendations and personalized visuals" (Chandrashekar et al., 2017).

For users, Netflix's approach to personalization is a key means of attachment to the platform. Personalization begins with splitting the presentation of content into compartments (that is, profiles and accounts). The distribution of content through profiles invites users to think of themselves as owners of content and to consider their consumption primarily from an individual perspective. Referring to his roommates, Antonio, a 23-year-old clerk at a public university, explained why he created his own profile: "it's not that we don't get along, or anything like that, it's just that Netflix is [a] very independent [thing]." Thus, the expression " $m y$ profile" or " $m y$ account" was commonly used to describe people's relationship with Netflix.

An important expression of personalization is the configuration of the platform's preferences, such as selecting Spanish or English as the preferred language in which to display content (including the names of series and movies) or adding specific images as avatars of users' profiles. The purpose here is twofold: to provide the platform with a feature of the user's personality and, at the same time, to help users perform an identity in relation to the platform. Figure 1 shows this dynamic: the four profiles of the account belong to different people, three of whom live in the same household. 


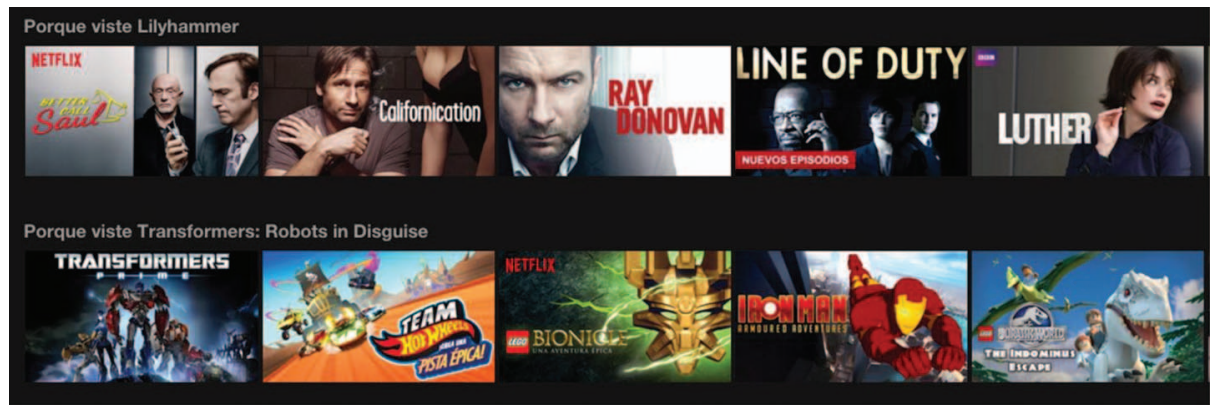

Figure 2 Recommendations based on content seen previously by Manuel.

As people use the platform more frequently, they intensify efforts to control aspects of their profiles. To make sense of this process, users typically employ the metaphor of contamination: content that was chosen by another person pollutes the personalization process. Lara, who is 26 years old, explains:

My sister and I have very different tastes. She saw some soap operas and some things that I thought were embarrassing when they appeared on my profile and it made me angry because [my] recommendations changed [as a result]. When I realized it, I said: "Please, get your own account and stop polluting my profile with things that I do not want!"

Characterizing the situation in terms of contamination (i.e., a problem) invites a concrete response from users (i.e., a solution): actions to clean up any disorder, including the creation of more profiles or using third-party accounts to watch content about which users are unsure and think could contaminate their profiles.

For users, personalization is the key to successful recommendations and, therefore, it must be protected with care. Thus, Sandra, a 24-year-old university student, explains: "it bothered me when [my sister] grabbed my profile because then I would get things that I didn't want to see, so I said, 'Get your own!'” This theory of how the algorithm works is common and is powered by the platform, which often displays the phrase, "because you watched ..." (see Figure 2). Users interpret this as a clue provided by the platform to learn how to customize their user experiences.

Personalization also occurs when users treat Netflix as an Other with which they can interact or have a sort of dialogue: inputs are provided to the platform (through practices such as consuming content, creating personal lists, evaluating shows and movies, etc.) and, in return, the platform offers personalized content, customized recommendations, popularity ratings, and comments from other users. In this sense, algorithms are what make Netflix a personal platform. Lara, for example, treated Netflix as a female counterpart who could provide personalized recommendations through the exchange of appropriate information: "I know she [ella] is trying to give me recommendations based on [my] taste; everything Netflix gives me will be based on my own consumption profile." Domesticating Netflix thus becomes an act of 
self-performance through a profile: a matter of giving the platform the right cues so she can interpret one's taste appropriately.

Personalization dynamics neatly illustrate what Vaidhyanathan $(2011$, p. 110) referred to as "infrastructural imperialism": that is, how platforms such as Netflix "structure ways of seeking, finding, exploring, buying, and presenting [data] that influence (though they do not control) habits of thought and action." This form of influence, Vaidhyanathan argued, is much more profound than traditional cultural imperialism, in that it helps to naturalize settings (such as the personal profile) as the default way of interpreting culture and the self. In this way, under the promise of getting an individual, unique version of the platform, users incorporate the precise mechanism through which Netflix also seeks to domesticate them: the segregation of consumption practices into compartments that allow the company to colonize them.

\section{Integration}

By integration, we refer to the combination of criteria that each person takes into consideration as they consider what content they want to watch. We found at least five criteria that are variously combined: interpersonal relationships, technical characteristics of the content, the role of Netflix as a producer of original content, reviews in specialized outlets, and opinions available in social media. Thus, algorithmic recommendations do not work entirely alone, nor do they act as the only determinant of consumption for Netflix users. Instead, users integrate algorithmic recommendations into a matrix of sources and criteria based on their sociocultural backgrounds and personal or professional interests.

A decisive criterion for some people are thoughts from opinion leaders: that is, people whose perspective they value and respect. Catalina, an audiovisual producer who runs her own company, pointed out: "the number of times I watch something that Netflix recommends to me is really tiny compared to the number of times I do it because someone else recommended it to me." Users who expressed a similar opinion attributed it to two related factors: neither Netflix could know them as well as the people around them, nor can they know Netflix as well as they know their opinion leaders. These users perceive Netflix as interested in providing recommendations for commercial reasons. Therefore, its suggestions should be viewed with suspicion.

Another factor that shapes the consumption of the platform is production characteristics, such as the leading actor or actress, members of the production team, or the presence of a director or cinematographer, among others. This factor appeared as a prevalent consideration in our sample of respondents, given that some of them work in the communications industry. Thus, the significance of this source of recommendations is conditioned by cultural and professional codes. Netflix makes it easy to implement this criterion by providing abundant information about each content characteristic available on the platform.

Some users also value Netflix's role in creating and developing original content. In short, they associate Netflix with the notion of quality and, thus, receive positively 


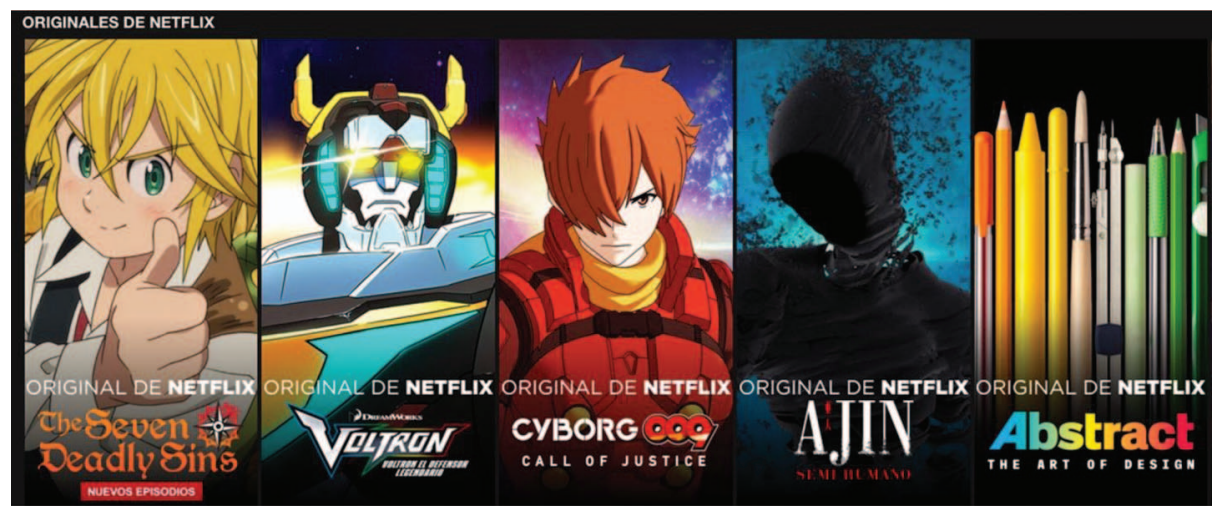

Figure 3 Netflix Originals displayed on Lara's profile.

any recommendation about its original productions. This imaginary emphasizes the value of the algorithmic model employed by the Netflix brand to produce and distribute content. Antonio, the clerk at a public university, maintained: "I feel that all the products that they [Netflix] do, they may not all be for the same audiences, but they are all very well made, very well designed, [and] there is a variety of content." Netflix displays this content in a specific section of the platform's interface, which includes the company's logo and the phrase "Netflix Originals." This section also shows large, personalized images, each show's logo, and a notification when new episodes are available (see Figure 3). In this way, it becomes easier for users to identify and consume these recommendations.

Reviews of films or series on specialized websites were also important for several interviewees. Irene, a 20-year-old advertising student, said: "I try to read reviews [and], if I get good reviews of any movie, then I try to watch it." One factor that complicates the selection of content for several people is the abundance of products to watch. For them, the expertise of reviewers can make a difference when choosing which content to consume on Netflix. However, this does not prevent Netflix from reaching users with specific recommendations. The company has indicated that it also incorporates "external data to improve [its algorithmic recommendation] features [...] such as box office performance or critic reviews" (Amatriain \& Basilico, 2012b).

Finally, some users employed social media to ask for recommendations. Sandra, the university student mentioned above, noted: "Sometimes I write on Facebook or Twitter: 'Hey, what series do you recommend on Netflix?'” Answers function as votes and as a way to assess criteria about specific recommendations. Whether this content was originally suggested to others by algorithms is usually unknown to users but, by asking their contacts, they feel these recommendations come from a trusted source. Social media also filter algorithmic recommendations through unsolicited comments about specific content that is gaining popularity. Rodrigo, a 26-year-old economist, says: "they [friends on Facebook] start[ed] talking a lot about 
series like Black Mirror, so [I] watch[ed] it." Thus, comments in social media also work as recommendations. Users like Rodrigo feel they must watch content that becomes popular in their social circles because, otherwise, they might be isolated. Furthermore, these comments create pressure to watch suggested content quickly, because there is always the possibility that others will spoil plot elements before the individual has had a chance to watch the content.

Users integrate these criteria differently into their lives and assign various weights to them, depending on a matrix of social, cultural, personal, and professional codes. In this way, they create their own repertoires and hierarchies of criteria. María, a 20 -year-old university student who primarily valued recommendations from trusted friends, explained why she considered watching content associated with a particular genre (British Movies) that was displayed on her profile: "my father is a fan of British cars, and so is my grandfather. Not only cars, but the culture as well, the machines. That is truly important in my life. I was shaped by this." A symbolic connection with members of her family thus enabled her to integrate algorithmic recommendations into her criteria, despite her initial resistance. Sofía, a 41-year-old university professor, said about romantic movies recommended to her: "sometimes I watch a movie that is awful [un bodrio], but I watch it from a gender studies perspective and later use it as an example [in class]." Thus, participation in certain social and professional spaces creates conditions for integrating algorithmic recommendations.

\section{Rituals}

The domestication of algorithmic recommendations is done through routines and events that take place in specific times and places. By rituals, we refer to the ways in which users incorporate Netflix into their mundane activities in a systematic way and create consumption practices around which temporal and spatial processes are organized. We draw on Couldry's (2003, p. 2) conception of rituals as "actions organised around key media-related categories and boundaries, whose performance ... helps legitimate the underlying 'value' expressed in the idea that the media is our access point to our social centre." Rituals on Netflix operate through a twofold process: they require adjusting daily life to watch content on the platform, as much as adapting Netflix for daily life activities. In turn, Netflix makes specific recommendations to shape these rituals, based on the technologies users employ and the content they watch when they perform the rituals.

We identified two types of ritual domestication of the platform. One kind of ritual centers on the individual domestication of Netflix. Users typically make the consumption of Netflix compatible with the most mundane activities of their daily lives. Netflix is consumed "when I eat," "when I wash clothes," or "when I'm getting ready." As with personalization dynamics, Netflix functions as an Other or companion. This is not unlike what audiences had previously done with television. Jimena, a 29-year-old freelance consultant for small organizations, explained: "I sit down for lunch and turn Netflix on. [I put] some show on ... . even if I have seen it a thousand times. I do it to 
have a companion. When I iron my clothes, I turn on Netflix." Netflix makes it easy to perform this ritual: it can be seen in different devices, at various places, and it can be interrupted and resumed without major alterations. Since Netflix can be so easily incorporated into the spatial and temporal conditions of daily life, watching content on the platform and carrying out daily chores need not be separated.

The ritual of watching Netflix individually also requires adjusting the platform to this end. Users look for specific content, such as shows, given that they are episodic (which helps the repetitive nature of the ritual) and shorter than movies (and thus make it easier to allocate time throughout the day). Although this kind of content was the most prevalently watched among our interviewees, individual rituals are not restricted to (short) episodes. Users also reported watching movies across various sessions throughout a day or week. Personal devices that are easier to carry around, such as cell phones, tablets, or personal computers, are usually preferred to perform this ritual. This also helps users to extend the domestication process outside of the household, such as in work offices, in education centers, or on public transportation.

Another kind of ritual characterizes the collective domestication of the platform. As with individual consumption, adjustments are required both to daily life, content, and technology in order to carry out this ritual. Collective rituals occur at preestablished times and involve other people. As Couldry (2003) noted, rituals rely on the establishment of boundaries: a form of social interaction is created around Netflix by deciding who gets to participate in them. Rituals matter because they represent a means to define ways of being social. This is the value that underlies the performance of rituals, according to Couldry. The purpose of collective rituals thus transcends entertainment and focuses on interpersonal connections with members of the family, partners, or friends. Monica, a 24-year-old woman who works in marketing, said: "it's the Saturday movies that I watch with my mom and my sister. I have to look for something that all of us like, which is a bit [un toque] difficult." For the most part, users turn to television (the artifact) to perform this ritual because the screen is larger and has better sound than small devices. The most common content for this ritual are movies (which have a specific duration and narrative structures that help to bound the ritual temporally), but users can also select shows and watch (or binge watch) several episodes.

Algorithmic recommendations are central in consolidating the notion of Netflix as the center of users' lives through rituals. The platform employs several mechanisms to domesticate users through algorithms. By tracking "the device that the member is using" and "the time of day and the day of week" in which rituals take place, Netflix can adjust recommendations to suggest certain kinds of content and, thus, shape these rituals (Chandrashekar et al., 2017). The platform also uses genres to recommend specific content. Users typically employ genres recommended by Netflix as an instrument of consensus (see Figure 4). This was practically the only instance in which users seemed to assign some value to traditional audiovisual genres. Monica explained: "I do see [the main genre categories] one by one when I have to watch it 


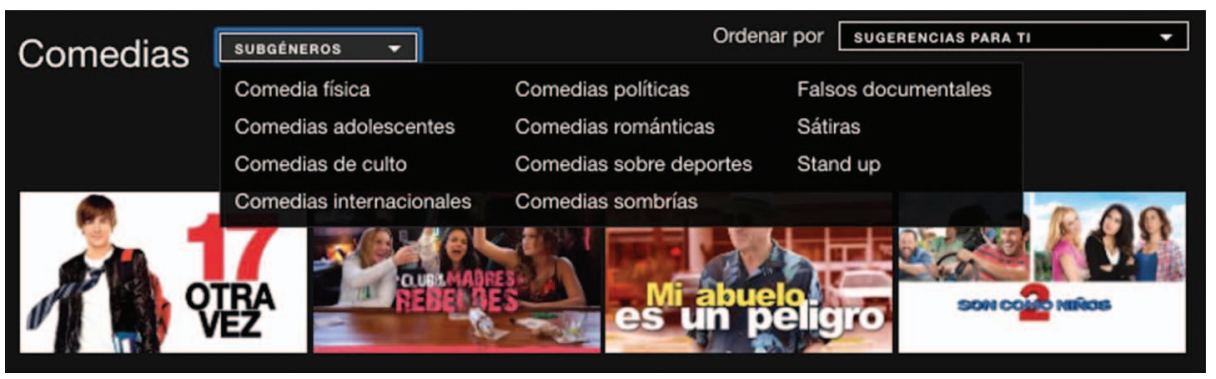

Figure 4 Sub-categories of the comedy genre recommended to Antonio.

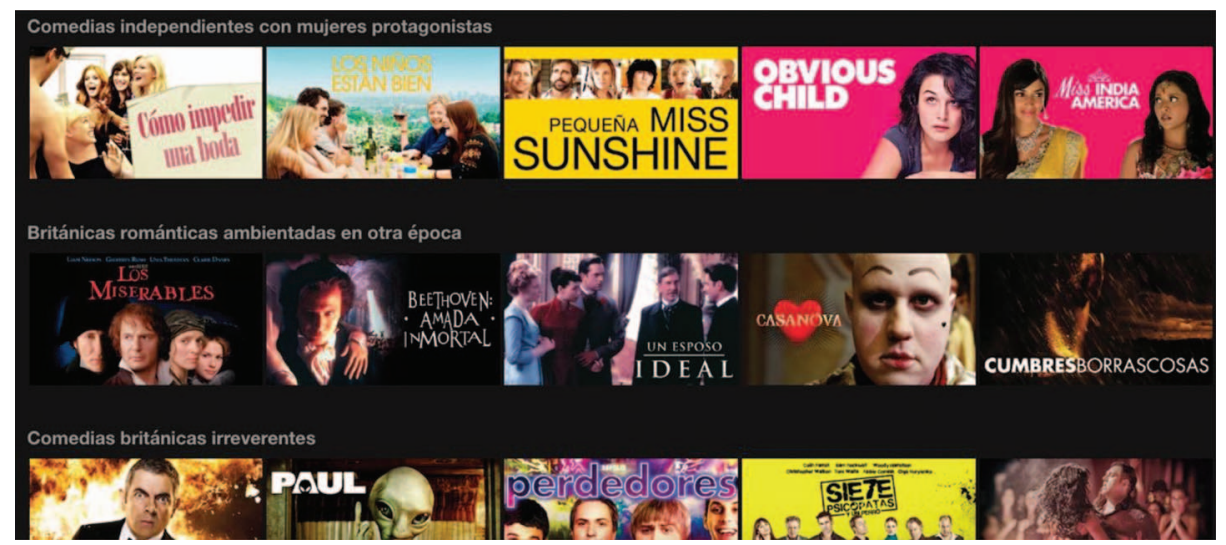

Figure 5 Alternative genres recommended to Sofía.

with my mom. I have to go through them one by one to [decide] what [we want to watch]."

A particular variation of this pattern are so-called "alternative genres" (see Figure 5). Through these recommendations, some users confirm that personalization mechanisms have worked. Sofía, the university professor, commented on an alternative genre that was recommended to her: "regarding shows, they [Netflix] know me inside out! It's become [a] pattern, because I do watch shows with 'Strong, Police Women." In this way, an alternative genre recommended to a user becomes a marker of identity. At the same time, some users showed surprise and dissatisfaction with the specificity and apparent arbitrariness of the genres created by Netflix. "It never occurred to me to think of this as a category," said Julieta, 25 years old.

The prevalence of rituals reveals the continued relevance of Silverstone's work. The processes of objectification and incorporation remain highly relevant: domesticating Netflix is performed at certain times of the day and requires locating certain technologies in specific places. It is precisely through these rituals that users naturalize the 
notion of Netflix as a social center. In other words, through individual and collective rituals, users act out the centrality of Netflix in their daily life (Couldry, 2003).

\section{Resistance}

Although interviewees widely appreciate Netflix, they also criticize some of its aspects. This permeates the relationship with recommendation algorithms and, thus, ends up shaping the mutual domestication process. We refer to these critical conceptions as resistance.

A source of resistance stems from the opacity of the platform. As noted previously, users constantly try to understand how the platform and its algorithms work. Jorge, a 34-year-old Master's degree student, explained his own theory thusly: "what I think is that [Netflix] makes recommendations based on what I've already seen." However, the lack of clarity on how users receive specific recommendations (and not others) generates some frustration from users. Jorge elaborated: "suddenly, they throw [suggest] things that make you wonder: 'How did I get this? Why do you suggest this?' ... Some recommendations do not fit with what I watched [previously]." Jorge thus felt surprised by recommendation criterion that remained incomprehensible or incomplete.

In other cases, resistance derives from what people interpret as a misreading of the inputs given to Netflix through consumption practices. Adriana, a public relations specialist and business administration student, maintained: "sometimes it's completely wrong [se la pela] with the recommendations and I [ask myself]: 'How can Netflix not know me at this point?'” Adriana's reflections prompt two observations. First, users expect concrete results after investing time and efforts in personalization dynamics. Second, although how Netflix operates is never fully understood, the expectations of the recommendation mechanisms are high: users expect that Netflix (as an Other) will capture not only their tastes but also their personalities. Algorithmic recommendations are seen as the proof that this has occurred, or not.

Users resist not only functional and technical aspects, but also the cultural biases inscribed in Netflix's recommendations. These biases are expressed in the constant recommendation of content that users consider stereotypical. Thus, Julieta, a 25year-old audiovisual producer, complained during the interview: "one went into the 'Latino' catalog and there were only telenovelas." Algorithmic biases become evident both in the content that is available and that which is not recommended. Fernando, a 22-year-old lesbian, gay, bisexual, transgender, and intersex (LGBTI) rights activist, explained: "the LGBTI category is nowhere to be found. ... That category does exist. But Netflix never gives [it to] me, for some strange reason.” This assertion reveals again the high expectations placed on the platform and the frustration generated by its opacity.

A widespread criticism among our interviewees was the lack of certain content in Netflix's catalog for Latin America, which was manifested in content that was 
not recommended to them. Users assumed that there was a singular catalog for Latin American countries. Many disputed the criteria employed to restrict content to countries of the region, including Costa Rica. Adriana expressed this discomfort with precision: "if there is something that truly bothers me, it's the fact that the Latin American catalog is completely different from the US catalog. It bothers me that they have Latin Americans pegged as 'not class A' clients." A quality distinction was thus made between content offered in the United States and products available in Latin America. This dissatisfaction centered on a discrepancy that users deemed arbitrary: although they paid exactly the same prices, they didn't have access to the same content available in the United States.

Netflix's catalog in Costa Rica is almost 30\% smaller than in the United States (Moody, 2018). But the complaint was not entirely based on quantitative criteria. This form of resistance offers cues to better understand claims of cultural imperialism. Since 2015, Netflix has produced original content in countries of Latin America with large local production industries and vast consumer markets (notably Brazil, Colombia, and Mexico). The platform has almost no content produced in smaller Latin American countries, such as Costa Rica (except for a few films). Although interviewees claimed to have watched (and appreciated) Netflix's Latin American original productions, they didn't always consider them as an alternative to content uniquely available in the United States. They valued English-speaking content because it allowed them to be a part of global conversations spurred by Netflix. Thus, they interpreted catalog differences as a form of exclusion. This partially complicates the notion that the North-South division doesn't matter in "data colonialism" (Couldry \& Mejias, 2019).

Despite the criticism, resistance did not translate into attempts to abandon Netflix. As noted previously, many interviewees continued to watch content through torrent applications (even those who paid for Netflix), where some "Netflix Originals" could be found. Moreover, users relativized their criticisms before giving up on the platform. The only reason that led to cancelling a subscription among our informants was the lack of financial resources after particular situations (such as layoff or change of work). Some users (although a minority) claimed to have employed Virtual private network (VPN) applications to reflect Internet protocol (IP) addresses in the United States or England and, thus, watch content available in those countries. Netflix evinced once again its desire to domesticate users, not only by forbidding this practice and temporarily blocking the operation of VPNs, but also by using resistance as a mechanism to improve the profiling of its users.

Acts of resistance may be interpreted as an expression of agency (Siles \& Boczkowski, 2012). Thus, examining resistance matters because it helps to downplay current "[reassertions of] monolithic accounts of power that tend to downplay or exclude audiences and the significance of the lifeworld" in datification processes (Livingstone, 2019, p. 171). For example, Manuel, a 36-year-old computer scientist, explained how his domestication of Netflix with his wife led him to a certain kind of awareness, freedom, and control: 
It generates the feeling of being aware of other types of things: we no longer depend on a schedule, so I spend more time looking for something; we see it together; we are not [under] the dictatorship of programming. We like that a lot. If we fall asleep watching something, we simply repeat it the next day, or if we're very anxious about [watching] something, then we watch immediately.

Other users offered similar accounts of empowerment through the use of Netflix. Yet the agency enacted in appropriating algorithms should be situated within the tensions that underlie the process of mutual domestication. It is difficult to determine how much of this sense of agency is also designed and engineered by Netflix to domesticate users. The company has consistently emphasized the need to promote a sense of awareness in users in order to motivate certain consumer behaviors. According to the company's technology blog:

[An] important element in Netflix' personalization is awareness. We want members to be aware of how we are adapting to their tastes. This not only promotes trust in the system, but encourages members to give feedback that will result in better recommendations. (Amatriain \& Basilico, 2012a).

For Netflix, a reflexive sense of agency in users does not necessarily predate the domestication of the platform, but is also a product of the work of algorithms.

\section{Conversion}

By conversion, we refer to the set of mechanisms employed by users to transform their private consumption of the platform into a public issue. Conversion is the process of reconnecting with the public world through the display of the technology or its contents. For Silverstone (1994), conversion turns media technologies into both the cause and product of conversations with others.

Conversion is key for users, in that it helps them build identities and establish interpersonal relationships. Manuel framed conversion as a contribution to others and, thus, rendered it indispensable:

There is so much content available [that] it can be difficult to find things of good quality. Thus, when I find something that I consider valuable, I share it. If [my wife and I] find something and it seems good to us, it goes [immediately] to Facebook.

These comments bring together conversion and integration dynamics. They reveal a desire to transform knowledge of the platform into an opportunity to influence others in their decision process. In this way, Netflix becomes a fundamental piece in the definition of users' tastes.

Through conversion, Netflix gets embedded in a wider digital ecology. Users employ various technologies to discuss content with other people, including cell phones, social media, and messaging applications. Many use Facebook and Twitter to this end. This further complicates the formation of algorithmic cultures, in that these discussions and recommendations get furthered filtered by the algorithms on which other technologies rely. Silverstone (1994) tied conversion to the interrelated issues of status, belonging, and competence. These issues are exemplified by the case 
of Adriana, the public relations specialist mentioned previously. She used WhatsApp to discuss Netflix content with a group of friends located in various parts of the world. In her words:

I have a group on WhatsApp. We are approximately 10 from different countries: Costa Rica, Mexico, Argentina, Chile, Spain, Bolivia, Ecuador, Venezuela, and Colombia. And Netflix is the same for everyone, except for the Spaniard. ... We share many contents. One of us will say: "I watched this show and thought you would like it." If I watched something it on Netflix and I liked it, I bring the subject to the group and discuss it.

In this example, Netflix is a shared object of attention across Latin America (and Europe). Relatively similar catalogs enable conversations based on shared cultural references. This attention to the platform becomes a way to signal membership in social groups that are relevant to users for both personal and professional reasons (in the case of Adriana, an online group of women interested in writing). As a professional in the field of communications, knowledge of specific content on Netflix and the capacity to recommend it is also associated with status. This creates fertile grounds for following algorithmic recommendations: they can provide a sort of capital for conversion dynamics.

Conversion is also infused with affect. María, the 20-year-old university student, who was watching a particular show by the time we interviewed her, noted: "I feel like I'm watching it because I love them [certain friends] so much that [I say to myself]: 'I'm going to watch it just to have something to talk about together."' The emotional value of associating with certain people (in this case, a group of girlfriends) motivated María to incorporate certain recommendations. Thus, conversion could be considered a fundamental part of media rituals, through which affective bonds are enacted and rendered meaningful (Couldry, 2003).

In turn, Netflix has consistently sought to use conversion dynamics on social media as a means to domesticate users. A first example was "Friends," an early initiative that allowed users to view each other's lists and queues and make recommendations, among other features. In 2013, the company implemented "Netflix Social," which enabled users to find out on Facebook what users were watching on Netflix. These examples make it obvious how much Netflix values "social data [as a] source of personalization" and its plans to use this information to algorithmically "process what connected friends ha[ve] watched or rated" (Amatriain \& Basilico, 2012b).

These initiatives have had relatively low success. In the media, Netflix has tended to blame users for resisting this form of domestication. In the words of the company's Chief Product Officer(CPO), "we have had ... major attempts at it and none of them have worked well. ... It's unfortunate because I think there's a lot of value in supplementing the algorithmic suggestions with personal suggestions" (Hunt, cited in McAlone, 2016). Similarly, Business Insider concluded that, "it's your fault Netflix doesn't have good social features" (McAlone, 2016). Users seem largely unconvinced that employing these features is of value in improving the recommendations they 
get. Thus, they turn to other technologies, which are much better integrated into their networks of interpersonal relationships, to this end. Perhaps arriving at a similar conclusion, Netflix's current strategy is to offer relatively generic features that allow users to share content from mobile apps.

Conversion reveals how users understand themselves as social beings in relation to Netflix. It shows how audiences incorporate the platform and its algorithms into the networks of interpersonal relationships, where their status, sense of belonging, and affect are partially defined.

\section{Algorithmic cultures and algorithms as culture}

By examining how users relate to Netflix's recommendation algorithms, this paper revisited one of the most influential concepts in the study of media technologies over the last 30 years. This exercise proved fruitful in revealing the relevance of Silverstone's work. Despite important transformations in television's "double articulation," the notion of domestication continues to provide an indispensable conceptual framework for understanding how media technologies are sold, acquired, imagined, placed, appropriated, and discussed in daily life.

We also sought to extend the theory of domestication to account for major transformations in Internet-distributed television. We posited the notion of mutual domestication to capture a parallel movement: how users incorporate Netflix into their daily life and how Netflix seeks to colonize users and turn them into ideal consumers through recommendation algorithms. This poses a challenge to dominant understandings of agency in domestication theory. In his analysis of the history of the concept, Silverstone (2006, p. 231, emphasis added) noted that the domestication approach was born as a reaction to technological determinism: "domestication was something human beings did to enhance and secure their everyday lives." To supplement this position, we argued that contemporary analyses of domestication must also recognize algorithms as actors that participate in how users get a certain sense of awareness, reflexivity, and control of their own practices. In other words, this requires focusing on how humans are increasingly targeted as subjects of domestication and data colonialism, in addition to how media technologies are objects of domestication.

Silverstone (1994, p. 124) emphasized the need to conceptualize domestication as "cyclical and dialectical." He elaborated: "consumption must be seen as a cycle, in which the dependent moments of consumption ... themselves feed back ... to influence and ... to define the structure and the pattern of commodification itself" (Silverstone, 1994, p. 124). He evoked the figure of a spiral to further explain domestication as a "dialectical movement" (Silverstone, 1994, p. 124). This idea applies neatly to the case of mutual domestication: its dynamics must be seen as interdependent, porous, and coconstitutive.

For example, efforts to personalize Netflix create expectations for other domestication dynamics. When these expectations are not met, resistance arises: users assume that Netflix should know them after they have revealed so much of themselves. 
Personalization also shapes the performance of rituals. Users expect that the "dialogue" they have established with the platform will serve as a basis for making decisions about which content can be watched individually or collectively. In turn, performing certain rituals can also lead to the conviction that it is necessary to open a personal account or profile.

Similarly, domestication relies heavily on conversion dynamics. Since the platform does not advertise in traditional media in the country (although the platform sponsors posts on social media), users have integrated the work of externalizing public opinions as part of domestication routines. Personal recommendations were a key criterion for many who decided to open a personal account or profile. People who valued recommendations from other users also felt it was important to filter algorithmic recommendations. In turn, integrating various recommendation sources triggers conversion dynamics. Likewise, acts of resistance can shape other dynamics of domestication. The frustration with the lack of certain content in Latin America led to new rituals, such as accessing Netflix through VPN. This, in turn, allowed users to implement recommendations offered by specialized media outlets about content not available in Costa Rica and launched new, personalized recommendations. Resistance thus triggers new ways to enact algorithms.

Silverstone also emphasized the cyclical nature of domestication as a theoretical intervention. He posited cyclicity to counterbalance two dominant theoretical extremes: on the one hand, "the overdetermination of the cultural industry which members of the Frankfurt School insist upon" and, on the other, "the kind of romanticism embodied in [the] phrase 'the actual brilliance' of the consumer" (Silverstone, 1994, p. 124). In this way, Silverstone also helped to balance out a similar dichotomy in which current discussions about datification and the role of algorithms take place. Following Silverstone, we argue that the notion of mutual domestication helps to account for two cyclical, parallel processes: on the one hand, cultures are algorithmic; on the other, algorithms are culture.

The intermediation of algorithms in processes of media consumption is shaping contemporary cultures. For users, algorithmic recommendations condition how they interact with the Netflix "culture machine" and how they make sense of its content. Users take Netflix's "infrastructural imperialism" as a given of the digital age. In this way, they assume that individual profiles and accounts are the default mode for relating to the platform. Users also naturalize profiling dynamics by framing their consumption practices as part of a "dialogue" established with an Other in order to get accurate recommendations. It thus becomes natural (and even beneficial) to use the platform's features to indicate their individual preferences. An algorithmic imaginary is idealized when users define Netflix - and the values on which it produces, markets, distributes, and recommends content-as the new standard of quality in contemporary cultural production. As such, it warrants loyalty from users, despite criticisms; it deserves being considered as a building block for self-performance and the maintenance of social relations. Algorithms thus play a central role in the rise of specific modes of cultural appropriation. 
Although it captures important aspects of the practices of Netflix users, the term "algorithmic culture" can also be misleading. Cultures are multidimensional and, as such, the ways in which people relate to media and their contents are conditioned by more than technology. Thus, mutual domestication also requires considering how algorithms are cultures in themselves: they are enacted through practices and rituals; their influence is shaped by users' social, cultural, and professional codes; and they are resisted and opposed. That is how they acquire meaning and are integrated into daily life. How people build repertoires of criteria for considering what content to watch is also the product of culturally situated processes. The very notion of personalization builds on the cultural history of individualism.

The study of algorithmic cultures and algorithms as cultures has been framed in oppositional terms (Seaver, 2017). By positing mutual domestication as cyclical, we argue that instead, when the use of media technologies is examined empirically, both processes are in constant interaction. ${ }^{1}$ They are simultaneous rather than sequential: users enact algorithmic recommendations as they incorporate them into their daily lives, but these algorithms are designed to adjust to these enactments in order to colonize users. It is precisely in this cyclical interaction that current debates about the role of algorithms in redefining, standardizing, or enriching culture must be situated.

\section{Notes}

1 How different enactments of technology enter into conflict or not should be a product of empirical investigation rather than an a priori decision. Recent work has shown that different enactments of realities or objects can coexist (Siles, 2013).

\section{References}

Amatriain, X., \& Basilico, J. (2012a). Netflix recommendations: Beyond the 5 stars (Part 1). The Netflix Tech Blog. Retrieved from https://medium.com/netflix-techblog/netflixrecommendations-beyond-the-5-stars-part-1-55838468f429

Amatriain, X., \& Basilico, J. (2012b). Netflix recommendations: Beyond the 5 stars (Part 2). The Netflix Technology Blog. Retrieved from https://medium.com/netflix-techblog/netflixrecommendations-beyond-the-5-stars-part-2-d9b96aa399f5

Bucher, T. (2017). The algorithmic imaginary: Exploring the ordinary affects of Facebook algorithms. Information, Communication \& Society, 20(1), 30-44. doi:10.1080/1369118X.2016.1154086.

Chandrashekar, A., Amat, F., Basilico, J., \& Jebara, T. (2017). Artwork personalization at Netflix. The Netflix Tech Blog. Retrieved from https://medium.com/netflix-techblog/artworkpersonalization-c589f074ad76

Corbin, J. M., \& Strauss, A. L. (2008). Basics of qualitative research: Techniques and procedures for developing grounded theory (3rd ed.). Thousand Oaks, CA: Sage. doi: $10.4135 / 9781452230153$.

Couldry, N. (2003). Media rituals. London, England: Routledge. 
Couldry, N., \& Mejias, U. A. (2019). Data colonialism: Rethinking big data's relation to the contemporary subject. Television \& New Media, 20(4): 336-349. doi: $10.1177 / 1527476418796632$.

Eslami, M., Rickman, A., Vaccaro, K., Aleyasen, A., Vuong, A., Karahalios, K., \& Sandvig, C. (2015). "I always assumed that I wasn't really that close to [her]": Reasoning about invisible algorithms in the news feed. New York, NY: Paper presented at the Annual Special Interest Group on Computer-Human Interaction, Association for Computing Machinery. The conference took place in April. Conference on Human Factors in Computing Systems.

Finn, E. (2017). What algorithms want: Imagination in the age of computing. Cambridge, MA: MIT Press. doi:10.7551/mitpress/9780262035927.001.0001.

Gao, G. (2015). Latin America's middle class grows, but in some regions more than others. Pew Research Center. Retrieved from http://www.pewresearch.org/fact-tank/2015/07/20/ latin-americas-middle-class-grows-but-in-some-regions-more-than-others/

Livingstone, S. (2018). Audiences in an age of datafication: Critical questions for media research. Television \& New Media, 20(2), 170-183. doi: 10.1177/1527476418811118.

Lobato, R. (2019). Netflix nations: The geography of digital distribution. New York, NY: New York University Press.

McAlone, N. (2016). It's your fault Netflix doesn't have good social features. Business Insider. Retrieved from http://www.businessinsider.com/netflix-users-dont-want-socialfeatures-2016-2

Moody, R. (2018). Which countries pay the most and least for Netflix? Comparitech. Retrieved from https://www.comparitech.com/blog/vpn-privacy/countries-netflix-cost/

Red 506 (2018). Red 506. San José, CA: El Financiero.

Rohit, P. (2014). Personalization, not House of Cards, is Netflix brand. WestSideToday.com. Retrieved from https://westsidetoday.com/2014/06/17/personalization-house-cardsnetflix-brand/

Seaver, N. (2017). Algorithms as culture: Some tactics for the ethnography of algorithmic systems. Big Data \& Society, 4(2), 1-12.

Siles, I. (2013). Inventing Twitter: An iterative approach to new media development. International Journal of Communication, 7, 2105-2127.

Siles, I., \& Boczkowski, P. J. (2012). At the intersection of content and materiality: A textomaterial perspective on agency in the use of media technologies. Communication Theory, 22(3), 227-249. doi:10.1111/j.1468-2885.2012.01408.x.

Silverstone, R. (1994). Television and everyday life. London, England: Routledge.

Silverstone, R. (2006). Domesticating domestication: Reflections on the life of a concept. In T. Berker, M. Hartmann, Y. Punie \& K. Ward (Eds.), Domestication of media and technology (pp. 229-248). Maidenhead Berkshire, UK.: Open University Press.

Vaidhyanathan, S. (2011). The Googlization of everything: (And why we should worry). Berkeley, CA: University of California Press.

Williams, R. (2001). The long revolution. Peterborough, Canada: Broadview Press. (Original work published in 1961.) 